http://jmscr.igmpublication.org/home/ ISSN (e)-2347-176x ISSN (p) 2455-0450 crossref DOI: https://dx.doi.org/10.18535/jmscr/v8i6.88

\author{
Journal Of Medical Science And Clinical Research \\ IGM Publication \\ An Official Publication of IGM Publication
}

\title{
Management of Hydatid Liver Disease in an Alcoholic Patient with Alcoholic Hepatitis and Alcohol Induced Pancreatitis- A Case Report Study
}

\author{
Authors \\ Karan S. Thakur ${ }^{1}$, Purnima Patial ${ }^{2 *}$ \\ ${ }^{1}$ Department of Surgery ZH Mandi, H.P. \\ ${ }^{2}$ Department of Medicine ZH Mandi, H.P. \\ *Corresponding Author \\ Purnima Patial
}

\begin{abstract}
Hydatid cyst is a parasitic zoonotic disease of tapeworms. Most cases are found in rural populations of the world. It may affect multiple organs of humans. Liver is one among the common sites involved. Inappropriate treatment may lead fatal outcome. Management of such cases requires thorough knowledge of the microbiology of the disease, disease course and complications. As surgery provides the best treatment in hydatid disease, it becomes necessary for surgeons to have complete knowledge about the disease. Management becomes complicated when patient develops some other concurrent illness. This article deals with case study of such a case, in which patient with Hydatid liver disease had alcoholic hepatitis and later developed alcohol induced pancreatitis.

Keywords: Hydatid disease, Pancreatitis, hepatitis, albendazole.
\end{abstract}

\section{Introduction}

Hydatid disease also called echinococcosisis a parasitic disease of tapeworm Echinococcous. The disease occurs in most parts of the world. Most cases belong to rural and animal grazing populations. Initially this disease usually develops without symptoms. Liver and lungs are commonly affected. The disease may also spread to other organs like brain. Patient mostly develops symptoms according to the affected system. Humans are infected by ingestion of eggs of the worm by close contact of infected animals or from food/water that contains eggs.

Diagnosis is usually made by USG and CT. Hydatid serology may support the diagnosis. Albendazole for long term is used for conservative treatment but surgery remains the treatment of choice.

\section{Cause}

Hydatid disease is caused by a tapeworm parasite Echinoccocus. These are Platyhelminthes of class cestoda. E. granulosus is the most common species causing the disease in humans. It is present in the dog intestine, eggs are ingested by humans and pass in the portal blood to the liver.

\section{Life Cycle}

Carnivores like dogs are the definitive host. Intermediate hosts are sheep and cattle. Humans are accidental hosts and are dead end host. 


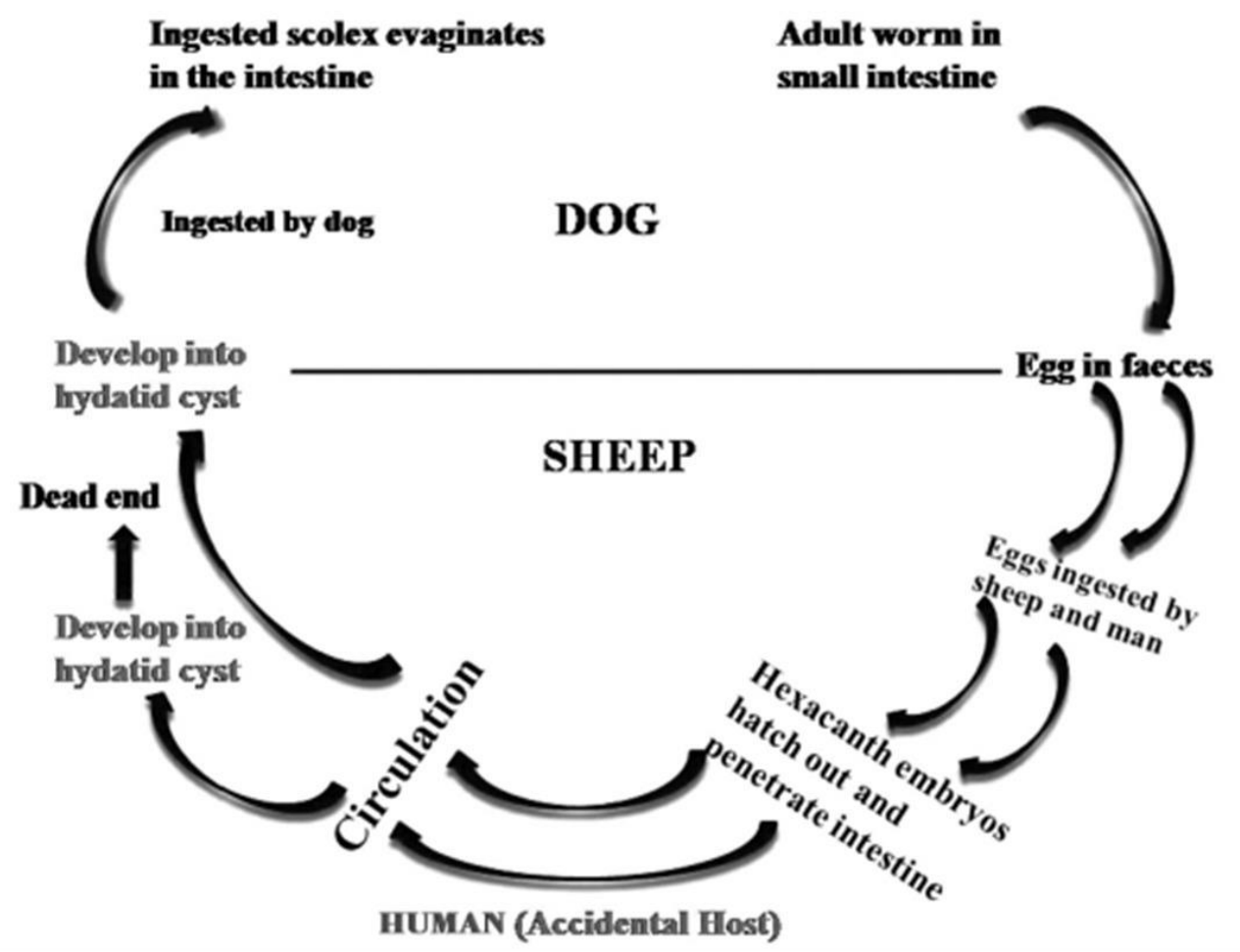

Figure 1: life cycle of Echinococcusgranulosus

\section{Clinical Features}

Generally disease is asymptomatic initially. Signs and symptoms depend on the site of location and size of the cyst. When liver is involved pain abdomen, weight loss, dyspepsia and jaundice may develop. Liver abscesses are often large by the time of presentation with upper abdominal discomfort or may present after minor abdominal trauma as an acute abdomen due to rupture of the cyst into the peritoneal cavity. Liver cysts can also rupture through the diaphragm producing an empyema, into the biliary tract producing obstructive jaundice, or into the stomach.

In lung hydatid chest pain, cough and shortness of breath may be the symptoms.

\section{Diagnosis}

Diagnosisof Hydatid disease may require a combination of radiological, serological, histopathological or nucleic acid detection techniques. Radiological imaging with USG is the first technique of choice, which is further supported by serological techniques. Diagnosis is suggested by the finding of a multiloculated cyst on ultrasound and is further supported by the finding of a floating membrane within the cysts on CT scan. CT and MRI may further aid in diagnosis. In addition to radiology and serology, PCR and histopathogy of biopsy are the alternate methods of diagnosis. In case of suspected biliary involvement ERCP or percutaneous transhepatic cholangiography may be necessary.

\section{Treatment}

Treatment is indicated to prevent progressive enlargement and rupture of the cysts. In the first instance, a course of albendazole or mebendazole may be tried. The treatment of choice in hydatid disease is surgery combined with chemotherapy. Albendazole or mebendazole are used as chemotherapy drugs before and after surgical removal of cysts. Most common form of surgical technique is open surgery. The surgical options range from liver resection or local excision of the cysts to de-roofing with evacuation of the contents. Alternatively laparoscopic surgery may be used.

For inoperable cases alternatives are long-term 


\section{JMSCR Vol||08||Issue||06||Page 458-463||June}

chemotherapy with albendazole or PAIR (puncture-aspiration-injection-reaspiration). PAIR should only be attempted if there is no communication with the biliary tree.

\section{Case Study}

A 34 year old male patient from a rural area, farmer by occupation presented in the department of medicine of our institution, in the month of December 2019, with complaints of pain abdomen, jaundice and abdominal discomfort. Liver function tests were deranged. Patient was chronic alcoholic and chronic smoker. Patient was evaluated and was diagnosed to have alcoholinduced hepatitis. Patient was treated for hepatitis. During the hospital course USG abdomen was done. USG showed large mass involving left lobe of liver measuring $7.2 \times 6.0 \times 7.2 \mathrm{~cm}$. Multiple small cysts seen mostly in the periphery region around central part. Possibility of hydatid cyst was kept. Hydatid serology was done, which came out to be positive. Patient managed medically for hepatitis. Patient was started on albendazole and surgical opinion taken.

Patient evaluated further from surgical department. CT abdomen was done. On CT a well defined rounded intraparenchymal cystic lesion seen in left lobe of liver involving segment 2 and 3. Multiple internal peripherally spaced daughter cyst and thin peripheral calcification seen. The lesion measured $65 \quad$ x $66 \quad$ x $59 \quad \mathrm{~mm}$. No abnormality was seen on Chest X-Ray. Patient was planned for surgery with chemotherapy both preoperative and postoperative.

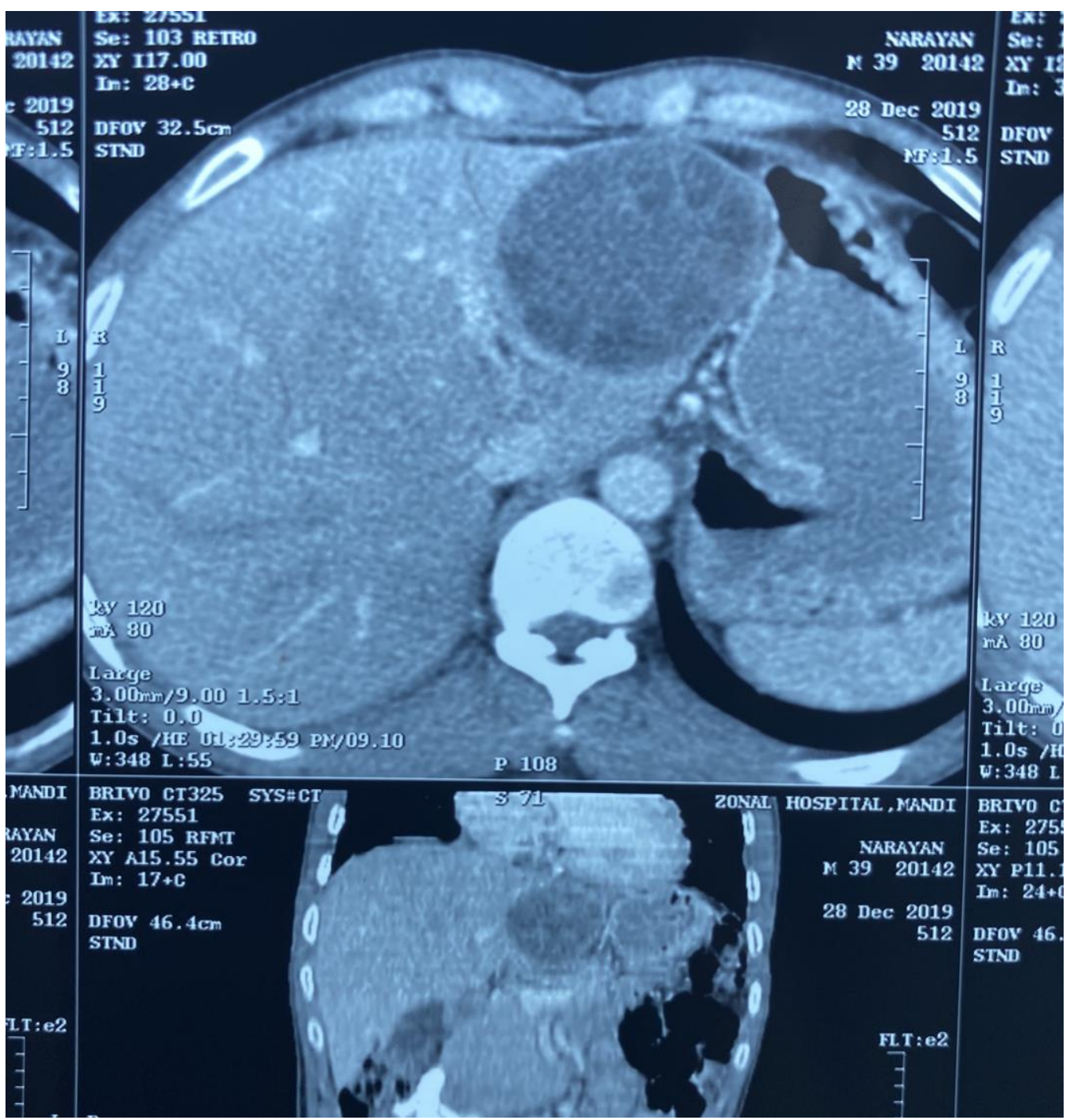

Figure 2: CT scan of the patient showing typical picture of Liver Hydatid cyst. Cyst is occupying the left lobe seen in both axial and coronal view. 


\section{JMSCR Vol||08||Issue||06||Page 458-463||June}

Patient was put on Albendazole for 2-3 months. But patient continued to consume alcohol, and developed pancreatitis during the course of chemotherapy. Patient was readmitted in February 2020 with alcohol induced acute pancreatitis. Patient was resuscitated. Pancreatitis was managed conservatively. Patient was discharged after acute pancreatitis resolved and albendazole was continued for 1 month. Patient was symptomatic during chemotherapy, had recurrent pain, abdominal discomfort. Patient was planned for surgery in the month of March.

Patient was admitted in surgical ward for elective surgery. All the necessary preoperative investigations were done. Anaethetists were informed about the risk of intraoperative anaphylaxis. Epinephrine and steroid were ready with anaesthetist. General anaesthesia was given. Midline incision given to open the abdomen. Abdomen explored, liver was mobilized. Cyst was exposed. Abdomen was completely packed off with mops soaked in scolicidal agent $10 \%$ povidone-iodine to prevent unwanted anaphylaxis and seeding in case of rupture and spillage of cyst. Cyst of size $\sim 8 \mathrm{~cm}$ occupying left lobe of liver was identified. Aspiration of cyst was done through a close suction system. Daughter cysts were aspirated; cyst was flushed with scolicidal agent. Cyst was then unroofed and contents were drained. Cyst was evacuated completely and assessed for any biliary communication. No cystobiliary communication found. No cyst content spillage occurred. Cyst was then irrigated and filled with scolicidal agent $10 \%$ povidoneiodine for 20 minutes. Partial cystectomy was done. The residual cavity space was reduced with Omentoplasty. Drain was kept in the cyst. Another drain was keptin subhepatic location. Packs were removed carefully. Abdomen was closed in layers.

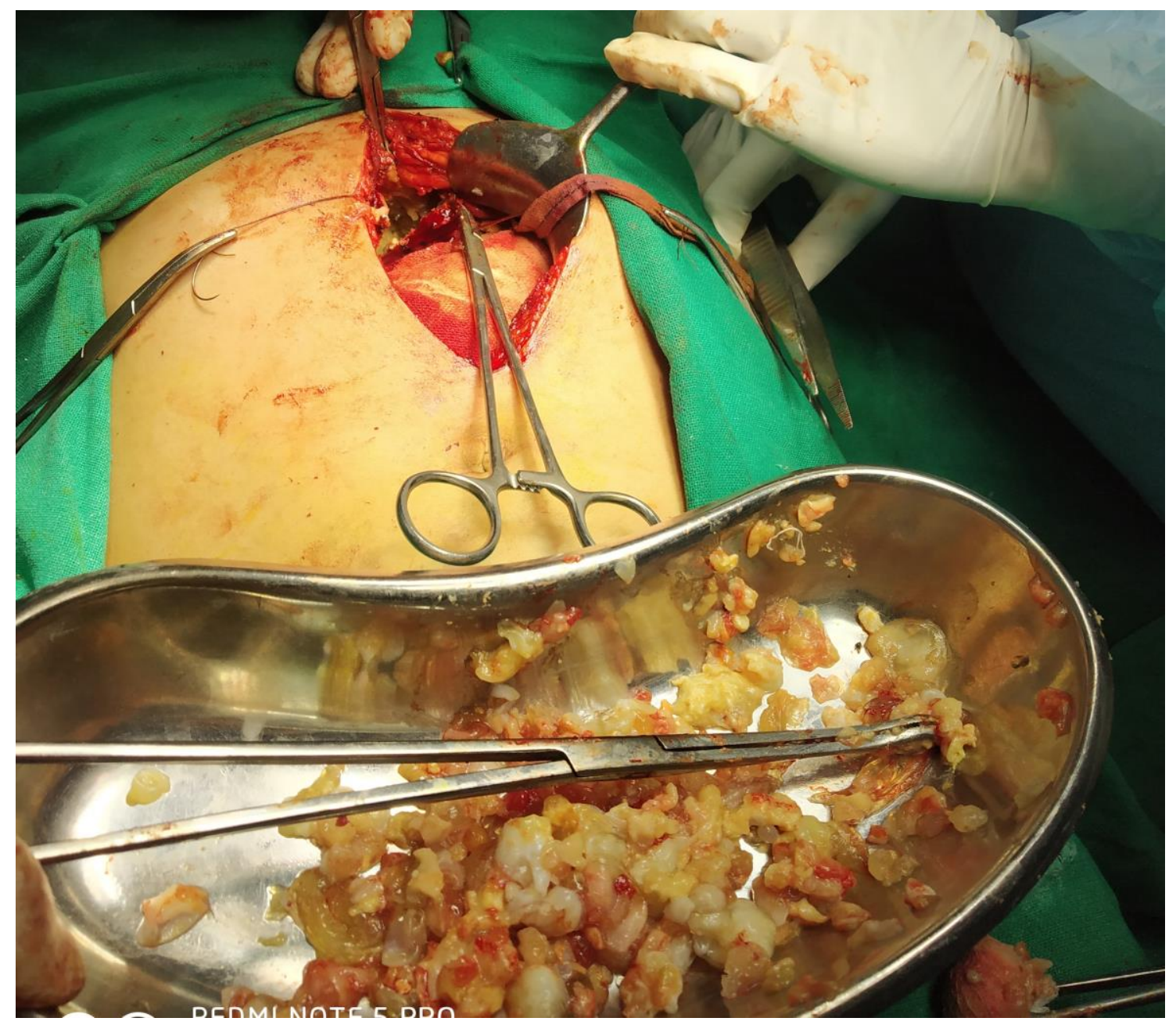

Figure 3: Intraoperative Cyst Exploration and removal of contents of cysts. 
Post-operative course was uneventful. Patient was discharged in a complete hemodynamic stable condition on post-operative day 5. Albendazole was continued for next 3 months. Patient was also advised not to consume alcohol. Follow-up was done regularly. Postoperative course during chemotherapy was uneventful. Patient was reassessed after 4 months of surgery. He had no symptoms and was cured completely.

\section{Observation and Result}

Management of hydatidcyst is complex. It becomes more challenging when patient develops other life threatening illnesses. In this case management became complex, as patient was chronic alcoholic. Initially he had alcoholic hepatitis, which was managed conservatively. Then chemotherapy was initiated. In such cases it is advisable to wait for hepatitis to get resolve, give a course of chemotherapy and then go for surgery.

As patient was planned for surgery, in between patient developed alcohol pancreatitis. Surgery was further delayed, and pancreatitis was managed first. So before going for surgery, pancreatitis which may be fatal should be managed early. Then surgery was performed. Both pre and post operative chemotherapy helped in good outcome of the patient. As albendazole was given preoperatively no intra-operative complications occurred. Similarly post operatively albendazole course was continued. Patient was cured completely.

Thus it is clear from the above case that surgery combined with chemotherapy is the best management for such patients.

\section{Discussion}

Hydatid disease is mainly a zoonotic parasitic disease of rural and animal grazing areas. In this case study management of hydatid disease in an alcoholic patient belonging to a poor socioeconomic status of a rural area is discussed.

Patient initially presented with symptoms of alcoholic hepatitis. On further evaluation patient was diagnosed to have hydatid disease. Hepatits was managed conservatively by medications. As open surgery plus chemotherapy is treatment of choice in hydatid disease, this patient was planned for elective surgery along with pre and post operative chemotherapy with albendazole. Patient was initially put on albendazole for 3 months before surgery. In the mean course patient developed alcohol induced pancreatitis. Patient was managed for pancreatitis. Open surgical removal of cyst was then done. Preoperative albendazole reduces intraoperative complications. This was followed by post operative albendazole for 3 months. Patient was cured completely. After 4 months on follow up patient was asymptomatic and was relieved completely.

So it is evident from the study that surgery along with chemotherapy is the mainstay oftreatement in hydatid disease. Chemotherapy with albendazole prevent spread of disease as well as post operative recurrences. Intraoperative complications like contamination of peritoneal cavity is also prevented by use of preoperative albendazole. In patients who develop concurrent life threatening complications like hepatitis/ pancreatitis, these should be treated appropriately before going for surgical intervention.

\section{References}

1. Norman SW, Christopher JK, Ronan P. The Liver, $27^{\text {th }}$ ed. Bailey and Love Short Practice of Surgery: 1153- 75

2. Smego R, Sebanego P. Treatment options for hepatic cystic echinococcosis. International Journal of Infectious Diseases. 2005;9:69-76.

3. Vagholkar K, Pawanarkar A, Vagholkar S et al.Surgical management of hepatic hydatid disease. Int Journal of Research in Med sciences. 2016 Jun;4(6):1834-37.

4. Anthony SF, Eugene B, Dennis LK et al. Cestode infections, 20 $0^{\text {th }}$ ed. Harrison's Principles of Internal Medicine: 1641-47. 
5. Anand S, Rajagopalan S, Mohan R. Management of liver hydatid cysts Current perspectives. Med J Armed Forces India 2012;68:304-9.

6. Courtney MT, Daniel B, Mark, E, Kenneth LM. The Liver, $20^{\text {th }}$ ed. Sabiston textbook of Surgery: 1452-55. 